\title{
La question laïque dans la France d'aujourd'hui Réflexions sur un passage de la norme à la valeur.
}

\author{
The secular question in France today. \\ Reflextions on a transition from norm to value.
}

Philippe Portier*

\begin{abstract}
Resumo
Este texto procura analisar o percurso da laicidade no decorrer das últimas décadas. $O$ que se desenvolve no âmbito da abordagem aqui proposta, assinala alguns dos deslocamentos, das mudanças e das transformações que se evidenciaram no contexto da laicidade francesa e europeia desde os anos 1980 até os dias atuais. Veremos como se dão as renovações e ressignificações teóricas que o termo laicidade adquire com o avanço do reconhecimento de novas reivindicações religiosas no quadro contemporâneo. O que se verifica entre outras coisas, é que podemos computar uma extensão considerável no que diz respeito ao reconhecimento das intervenções coletivas por parte das Igrejas e, ao mesmo tempo, registra-se o reforço das coesões que se firmam a partir das últimas décadas com a redescoberta da educação e da cidadania. Uma redescoberta que encontra aspirações renovadas em experiências sociais de ordem pública e jurídica. O que podemos notar no rol das pluralidades e das construções identitárias e religiosas atuais, é possivelmente a indicação de uma quarta era dos direitos humanos, uma era que incorpora os direitos culturais e que assiste ao retorno da religião no coração do debate social.
\end{abstract}

Palavras-chave: Laicidade ; contemporaneidade ; identidades religiosas

\begin{abstract}
This text intends to analyze the course of secularism over the last decades. What is developed on the ambit proposed here, points some of the movements, shifts and transformations that became evident on the French and European secularism context from the 1980s to the present day. A view of the theoretical renewal and meaning change that secularism term acquires with the progress of the recognition of new religious demands on the contemporary scenario will be given. What can be seen among other things is that we can estimate a remarkable extension concerning the collective intervention recognition by the church and, at the same time, there is a cohesion strengthening that have been established since the last decades with the rediscovery of education and citizenship. A rediscovery that finds renewed aspirations on social experiences of public and legal order. What can be noticed on the plurality and the current religious identity construction is possibly the suggestion of a fourth age of human rights, an age that takes on the cultural rights and assists the return of religion at the center of social debate.
\end{abstract}

Keywords: Secularism ; Contemporaneity; Religious identities

Article reçu 20 Février 2017 et approuvé le 4 mai 2017.

* Directeur de Groupe Societé, Religions et laicité (GSRL). Laboratórioligado a Ecole Pratique ds Hautes Etudes e ao CNRS e Directeur d'Etudes naEcole Pratique ds Hautes Etudes/Sorbonne. Pays d'origne: France. E-mail: philippe.portier@gsrl.cnrs.fr

Horizonte, Belo Horizonte, v. 15, n. 46, p. 443-456, abr./jun. 2017 - ISSN 2175-5841 


\section{Introduction}

La question laïque s'était retirée, depuis la fin de la Troisième République, de l'espace de nos délibérations collectives. Les fractures désormais se fixaient sur d'autres objets, économiques, institutionnels, internationaux. Elle ressurgissait parfois bien sûr. C'était cependant, comme en 1959 ou en 1984, à travers le prisme exclusif de l'École. D'évidence, la passion de la religion, dont Tocqueville rappelait volontiers qu'elle était dans ce pays "la première à s'allumer et la dernière à s'éteindre », ne faisait plus époque. Or, ces trois dernières décennies nous ont confrontés à une autre situation. Le retour de la revendication identitaire a nourri une réflexion renouvelée sur la laïcité, approchée désormais, non plus, comme du temps de nos passions scolaires, sous l'espèce d'un enjeu sectoriel, mais bien sous celui d'un objet à dimension universelle, renvoyant à la structuration globale de notre mode d'existence politique (PORTIER, 2016).

Un siècle après 1905, des discussions ont donc pris forme, et des textes bientôt sont venus, qui ont reconfiguré l'aménagement juridique de la place de la religion dans la société civile et dans les institutions publiques. Que s'est-il passé ? On peut décrire la mutation actuellement en cours comme le produit d'une réarticulation du rapport entre la norme et la valeur. Les valeurs renvoient à un modèle moral d'achèvement : «Elles expriment le caractère des biens considérés comme désirables par certains groupes de personnes ». Les normes se réfèrent, quant à elles, à un régime procédural d'organisation : elles désignent simplement les principes de base de l'ordre constitutionnel qui permettent aux citoyens d'être traités, de manière juste, en tant que « personnes libres et égales » (FERRY, 2002).

En son origine, la laïcité française s'agence de manière procédurale. Elle est, comme l'a noté Emile Poulat, un « instrument de pacification par le droit ». Il se fonde, dans le schéma que lui donne la loi de séparation du 9 décembre 1905, sur l'idée que chacun doit pouvoir, dans la sphère intime et sociale (ce qu'on appelle la « sphère privée »), cultiver et exprimer ses convictions et ses croyances comme bon lui semble, sous le surplomb d'un Etat parfaitement neutre à l'égard de toute 
conception du bien et, a fortiori, du salut ${ }^{1}$. Or, il semble bien que depuis une vingtaine d'années, nous soyons entrés subrepticement, en rupture avec le partage privé/public traditionnel, dans un autre régime de laïcité, où il s'agit de promouvoir au contraire «une culture commune exprimant ce que certains groupes de personnes considèrent comme étant préférable » (HABERMAS, 1987, p. 58): si l'on défend les droits des sujets croyants, et des institutions religieuses qui les accueillent, c'est en les subsumant, de plus en plus fortement, sous l'identité morale, souvent épaisse, que l'Etat entend non point seulement définir mais, également, imposer.

Cette « axiologisation de la laïcité », qui se traduit par une remise en cause de l'architecture séparative héritée du début du XXe siècle, est le produit d'une conjoncture inédite, marquée par un déplacement des enjeux, une reconfiguration des discours, une transformation des règles.

\section{Le déplacement des enjeux}

Si la question laïque s'efface dans les années 1960-1970 pour laisser place à un questionnement sur l' « hypothèse socialiste », c'est essentiellement parce que le religieux ne fait plus réellement problème : le catholicisme semble avoir rendu les armes, sous l'effet d'un double processus de sécularisation. Sécularisation interne, d'une part : depuis 1945, et plus encore, depuis les réflexions conciliaires qui ont intronisé la liberté religieuse, ses autorités épiscopales ont abandonné la théologie de l'Etat catholique, et reconnu la validité morale (et l'utilité pratique) du modèle de 1905. Sécularisation externe, d'autre part : l'Eglise est clairement alors dans une période de reflux. La pratique diminue, de même que les marques d'adhésion aux croyances et aux normes de l'institution. Les sociologues se font alors les confidents de la providence : la religion, annoncent-ils, est vouée à disparaître de notre champ d'expérience publique et même de notre horizon d'attente privé. Or, trente ans plus

\footnotetext{
${ }^{1}$ II ne s'agit là que d'une tendance. On sait l'importance de l'éducation sous la Troisième République. Cette politique scolaire n'empêche pas que les Eglises puissent, à partir de 1905, déterminer librement leurs principes d'organisation.
} 
tard, la donne n'est plus la même. Dieu fait retour au centre de nos préoccupations. S'agit-il d'un basculement total de la société dans le champ de la croyance ? Non. Il faut plutôt décrire la situation actuelle sous le registre de la « polarisation ».

Il apparaît, d'une part, que le mouvement de dissolution des appartenances religieuses, repéré par la sociologie de la sécularisation durant les décennies antérieures, s'est approfondi. La société française, à l’instar des autres sociétés européennes, persiste à se séparer de Dieu. Les déclarations d'appartenance ont fortement diminué. Dans les années 1950, les catholiques déclarés représentaient $92 \%$ de la population, les sans religion à peine 5\%. Les catholiques déclarés sont aujourd'hui aux alentours de 50\% ; les sans religion 40\%, dont la moitié se place dans la catégorie des athées convaincus. Ce déplacement s'accompagne d'une récusation des normes portées par les institutions ecclésiales. Souvent, ces sans religion sont plus ouverts aux appels de la spiritualité que les non-religieux des périodes antécédentes; ils cultivent même, parfois, la mémoire des « racines chrétiennes » de leur pays. Ils sont cependant, sur le terrain des options morales, foncièrement libéraux, ce qui peut expliquer leur résistance devant les attestations lourdes de religiosité en ce qu'elles leur semblent remettre en cause le principe d'autonomie dans lequel s'ancre leur imaginaire.

On relève, d'autre part, que d'autres secteurs de la population se sont réenracinés dans le religieux. Aucun des mondes confessionnels n'est à part de ce mouvement, ni les juifs avec le développement du courant ultra-orthodoxe ou la consolidation du courant orthodoxe, ni les protestants avec l'expansion des évangéliques, ni les catholiques comme on l'a vu par exemple lors de la controverse autour du mariage pour tous. Il affecte aussi l'univers musulman, et de manière bien plus visible parce qu'il tranche davantage avec les habitudes d'une société demeurée attachée à tout un corpus de gestes chrétiens. Ce revival musulman, dont on voit les indices, en particulier dans les jeunes générations mal intégrées, dès la fin des années 1980 (pratique de la prière et du ramadan, affichage des 
signes vestimentaires, puis respect des canons alimentaires), aurait pu ne s'exprimer que dans le cadre d'une piété domestique. Ce n'est pas ce qui est advenu. Il a fait l'objet d'une politisation. Comme pour effacer les stigmates du mépris social, les musulmans ont voulu obtenir de la part des pouvoirs publics une reconnaissance de leurs singularités, en n'hésitant pas à remettre en cause le partage traditionnel du privé et du public. Olivier Roy, dans son livre sur L’islam dans la République, résumait les choses de la manière suivante : «Les jeunes beurs des années 1980, quand ils sortaient de leurs banlieues, revendiquaient le discours dominant sur l'intégration, au lieu de défendre une différence, sinon dans la couleur de peau [...]. Ce qui est apparu plus tard, dans les années 1990, c'est un discours islamique structuré [...] qui fait [...] l'apologie d'une différence fondamentale, celle d'une croyance qui s'exhibe sans complexe » (ROY, 2005).

Cette requête de reconnaissance publique provoque très vite le débat, dès septembre 1989, à la faveur de l'affaire du voile à Creil. Deux raisons peuvent expliquer cette réactivité, si l'on excepte le fait que la discussion autour de l'« hypothèse socialiste » est désormais forclose. Une raison culturelle : la France révolutionnaire s'est fondée sur un « imaginaire de la généralité », insistant, « de manière très catholique » selon la formule de Tocqueville, sur ce qui unifie, plus que sur ce qui différencie. On n'oublie pas si aisément ce tropisme de l'universel, d'autant que le « problème » concerne là une population avec laquelle la France n’a pas réglé tous ses contentieux. Une raison sociale : s'était affirmée en France dans les années 1970 l'idée d'une pluralité heureuse, fondée sur l'harmonie naturelle des différences. Daniel Cohn-Bendit relevait très justement, il y a peu² : « J'ai connu une époque où l'on pouvait porter le voile, la kippa, la croix, et tout le monde s'en fichait ». Ce schéma est, dans les années 1990, frappé de décroyance. Comme le montrent la littérature des ministères (voir par exemple les rapports Delevoye ou Fragonard au milieu des années 1990) et les sondages d'opinion, la société française s'interroge alors, dans ce moment de l'histoire où l'Etat patriote démontre

${ }^{2}$ Dans une émission de télévision Des Paroles et des Actes du 26 janvier 2016. 
sans cesse davantage son impotence matérielle et symbolique, sur le devenir de sa cohésion interne. Cette angoisse de l'entropie est d'autant plus accentuée que la France est la victime de toute une série d'attentats commis au nom de l’islam, parfois même par des individus pourtant formés par l'école républicaine.

\section{La reconfiguration des discours}

Comment le régime républicain, qui est fondé sur le principe d'une séparation stricte entre le privé (où peut s'exprimer la différence) et le public (voué à l'expression du commun), peut-il accueillir ces revendications identitaires ? Cette question a suscité, à partir des années 1990, deux grandes séries de réponses dans la sphère intellectuelle. L'une, portée par des auteurs comme Michel Wieviorka ou Alain Touraine, entend défendre, même si le mot est d'usage récent, le principe d'une laïcité inclusive; l'autre, représentée par des auteurs comme Henri Pena Ruiz ou Catherine Kintzler, s'articule autour de la défense d'une laïcité universaliste. Ces polarités, qui acceptent bien sûr des différenciations internes, dessinent un espace de cadrage qui ont vocation à peser sur les politiques gouvernementales.

L’école de la « laïcité inclusive » propose un « multiculturalisme tempéré » pour parler comme Joël Roman (1996, p. 18-22). Il insiste clairement sur la nécessité de donner plus de poids à la reconnaissance des revendications religieuses et culturelles. Cette théorie regarde volontiers du côté du Canada, où la doctrine des « accommodements raisonnables », construite au début des années 1980, a permis d'accueillir le «droit à la différence ». Le fondement de cette position est double. Ses tenants, inspirés par Charles Taylor, insistent, d'une part, sur le fait qu'il est dans l'ordre de la liberté promu par une société libérale que chacun puisse exprimer ce qu'il ressent comme étant son identité profonde : la politique de l'égalité, née avec les Lumières, est appelée, par sa dynamique propre, à se déployer en politique de la singularité. Cette vision, ajoutent-ils, s'est d'ailleurs trouvée validée par toutes les institutions internationales lorsqu'elles ont intronisé 
la quatrième génération des droits de l'homme, celle des droits culturels. Dans ce schéma, le modèle de la République universaliste pose problème : s'il a pu être utile pour se défaire de l'âge ancien des hiérarchies, il définit aujourd'hui une politique de la discrimination en voulant instaurer la norme majoritaire en une norme nécessairement unanime, renvoyant elle-même à une forme de « communautarisme national ». Précisons d'emblée que ce différencialisme a ses limites: nos auteurs sont favorables certes à l'acceptation des demandes de reconnaissance, c'est à condition cependant que les individus puissent les porter devant l'Etat indépendamment de toute contrainte de leur groupe d'appartenance, et qu'elles ne remettent pas en cause les principes de liberté et d'égalité propres à l'ordre démo-libéral. Y a-t-il là un risque de rupture avec la communauté sociale dans son ensemble ? Loin de là. On fait valoir ici qu'en laissant les minorités qui le veulent exprimer publiquement leur identité, on favorise en fait leur intégration dans la société globale : la République, en tenant sa promesse d'égalité, leur donne le sentiment de les restaurer dans leur citoyenneté, prévenant ainsi les comportements d'exit psychique et politique. On n'opposera pas à cet argument le fait que les conduites d'affirmation sont le produit d'une contrainte externe. Il suffit de redonner la parole aux individus concernés : le signe religieux est souvent, pour eux, un moyen d'empowerment par lequel ils affirment tout à la fois leur dessein de se distancier des normes de leur milieu familial et de résister à un ordre global qui les marginalise. En outre, leur différencialisme n'est pas, contrairement à ce qu'on a pu dire, une forme de sécessionnisme : s'il fait fond sur une critique de la société telle qu'elle va, avec son matérialisme, son laïcisme, et parfois son racisme ordinaire, c'est généralement en espérant y trouver demain leur lieu de réalisation.

L'école de la « laïcité universaliste » entend bien, quant à elle, maintenir la séparation du privé et du public, non cependant sans donner du public une définition très extensive, ce que ne faisaient pas les fondateurs de la Troisième République. Ce schéma, qui aboutit à contenir la religion et parfois à la discipliner (en la soumettant à un juridictionnaliste concordataire), s'appuie sur des arguments symétriquement opposés à ceux avancés par les tenants de la thèse 
précédente. L'expression des identités religieuses dans l'espace public d'Etat pose un double problème, en dehors du fait que, d'importation anglo-saxonne, elle attente à la culture nationale. D’abord, elle peut mettre en péril la liberté de la personne, lorsque l'attitude religieuse est imposée par des proches de la personne concernée. On l'a vu à propos du voile. En 1995, Guy Coq, qui relève de ce courant, déclarait : « Le foulard peut agir comme pression insidieuse sur les jeunes filles traitées quotidiennement de mauvaises musulmanes »(QOC, 1995, p. 276). On évoque parfois le caractère aliénant en soi du signe, que ne rédime nullement le libre choix des femmes. Ce fut le cas, cette année, de la ministre des familles, Laurence Rossignol, lorsqu'avec une grande élégance, elle fit des femmes portant le voile, même de leur propre gré, les analogues des « nègres » qui consentaient, dans l'Amérique du XIXe siècle, à leur propre esclavage. Ce fut le propos aussi en 1989 d'Alain Finkielkraut lorsqu'il appelait à faire la « distinction entre les droits de l'homme et les droits des tribus ». Ensuite, le multiculturalisme peut mettre en péril aussi la cohésion de la nation en enfermant les individus dans leurs communautés d'origine, sans les ouvrir à l'altérité. On reconnaît là l'angoisse, si puissante depuis les années 1990, du «communautarisme ». Catherine Kintzler exprime parfaitement le point de vue de cette tendance dans l'extrait suivant : « La laïcité adjectivée (laïcité « plurielle », « ouverte », « positive », « raisonnable », « apaisée ») récuse le caractère neutre et minimaliste de la puissance publique républicaine, faisant de l'opinion religieuse une norme, autorisant les propos religieux au sein de l'État lui-même, et aboutissant à légitimer la communautarisation religieuse du corps politique »3. On est ici à fronts renversés par rapport au premier courant : la reconnaissance ne rassemble pas ; elle fracture. Ce discours-là a sa variante traditionaliste, du côté d'un Philippe de Villiers dès les années 1990, ou aujourd'hui du côté d'un Eric Zemmour ou des idéologues du Front national : ici on convoque la tradition chrétienne, et non la raison universelle, pour homogénéiser le commun. Ajoutons cependant que cette vision des choses ne peut être vraiment qualifiée de laïque, car elle introduit la possibilité

\footnotetext{
${ }^{3}$ Audition devant la Délégation aux droits des femmes du Sénat, 19 mars 2015.
} 
d’une inégalité évidente de traitement entre le monde judéo-chrétien et le monde musulman.

Au début des années 1990, la laïcité inclusive, telle qu’on vient de la décrire, a encore le vent en poupe. Sa situation s'est progressivement affaiblie. L'opinion publique, depuis le début des années 2000, est peu encline à aller dans le sens du libéralisme, d'autant que ses représentants se sont souvent recentrés sur un universalisme de combat, comme l'a montré, parmi d'autres textes, le rapport de François Baroin, significativement intitulé Pour une nouvelle laïcité, dans lequel il affirmait qu' «à un certain point la laïcité et les droits de l'homme sont incompatibles » ou, la même année, le rapport de la Commission Stasi qui dénonçait le risque du communautarisme. Cet ensemble de récits n’a pas été sans incident sur la configuration de la normativité juridique.

\section{La transformation des règles}

La loi du 9 décembre 1905 est certes, selon la formule du Conseil d'Etat, dans son rapport de 2004, la « clé de voûte » de la laïcité française. Le modèle qu'elle nous a légué n'est cependant pas demeuré dans une stabilité éternitaire. Quel est ce modèle des origines ? Il s'appuie sur la séparation entre le privé et le public. D’un côté, le public, identifié à la sphère de l'Etat, fait signe vers l'universel : chacun doit pouvoir se reconnaître en lui, qui représente le peuple dans son entier. De l'autre, le privé, qui ne se réduit pas à l'intime, mais intègre la société civile : là, les croyances peuvent s'affirmer librement dès lors que, dans l'espace externe du moins, chacun respecte l'ordre public. Or, au cours de la période récente, la France a opéré une remise en cause de cette dissociation fondatrice. S’est imposé un autre modèle, d'intrication celui-là, d'articulation du privé et du public : il s'est agi de renforcer les surveillances tout en consolidant les reconnaissances. L'aporie n'est qu'apparente: la reconnaissance ne vaut désormais qu'à l'intérieur d'un cadre normatif qui définit l'acceptable et justifie le contrôle. 
Le schéma de la reconnaissance s'était développé déjà dans les années 19601970. Il n'a guère été remis en cause depuis lors. Les financements constituent un pilier massif de ce point de vue. La République, contre ses règles initiales (loi Goblet de 1886, loi du 9 décembre 1905), les a développés : elle subventionne massivement les écoles privées depuis 1959, et accordent même des subventions indirectes aux associations cultuelles, ou aux associations ordinaires qui entreprennent de construire des édifices religieuses. Des autorisations d'absence peuvent être accordées aux fonctionnaires lors des grandes fêtes de leur religion, les cimetières accueillent des carrés confessionnels, des accommodements concernant les menus interviennent dans la restauration collective, les « besoins spirituels » des patients dans les hôpitaux sont de plus en plus pris en compte. L'ouverture des programmes scolaires à l'enseignement des faits religieux manifeste aussi une évolution de ce point de vue, d'autant qu'on la justifie, non point seulement par des motifs académiques, mais aussi par la nécessité de mieux connaître l'autre dans une société marquée par l'«accélération de la pluralisation ». Si l'Etat soutient les Eglises, il attend d'elles aussi qu'elles le soutiennent. Il leur demande, par exemple, d'assumer des fonctions d'expertise (notamment lorsqu'il s'agit de réfléchir à des questions émergentes qui ne renvoient plus simplement à la question du juste mais à celle du bien, ce qui est le cas dans le domaine de la bioéthique), ou des fonctions de médiation (comme on le voit lorsque les municipalités initient des opérations de dialogue inter-religieux ou inter-convictionnel). Cette évolution répond aux demandes d'une partie de la population : il est très difficile pour une démocratie libérale de les méconnaitre, d'autant que cette population est composée d'électeurs. Elle est un effet aussi de la crise du politique: l'Etat a besoin désormais des ressources cognitives et matérielles des Eglises. La reconnaissance du religieux ne peut être dissociée des impératifs de régulation de la société.

Le souci de la cohésion n'est pas vraiment à l'agenda du gouvernement dans les années 1970-1980. Comme on l'a relevé, il se manifeste puissamment, en revanche, dans les années 1990, selon une tendance qui n'a cessé de s'amplifier au 
cours des quinze dernières années. Cette inflexion intégrationniste, que l'on veut voir porter par l'idée de laïcité, s'est construite autour d'un dispositif de formation, ce dont témoigne la réactivation de l'enseignement civique et morale dans les écoles, mais aussi l'élaboration de diverses chartes de la laïcité, dans les administrations d'Etat, les hôpitaux, et, bien sûr, les écoles. Il faut aussi prendre en compte l'existence d'un dispositif de contention du religieux. Au cours de la période récente, les gouvernants, quelle que soit leur couleur politique, n’ont cessé, soutenus par la jurisprudence du Conseil d'Etat, de rappeler des règles antérieures - dont on ne faisait plus guère état - concernant la neutralité des fonctionnaires. Cela revient constamment, quelle que soit la couleur politique des gouvernements, soutenus par la jurisprudence du Conseil d'Etat. Mais il faut faire référence surtout aux lois de 2004 sur le port des signes religieux ostensibles à l'école publique et de 2010 sur la dissimulation du visage dans l'espace public. Ce mouvement juridique nous confronte à deux modifications essentielles du droit de la laïcité, même si leur mise en œuvre ne concerne au fond, quantitativement, que de petits nombres (PORTIER, 2016).

Le législateur a, d'une part, redéfini les espaces d'application de la règle de neutralité. Dans le modèle de 1905, seuls les espaces d'Etat étaient concernés par l'abstention religieuse, et encore pour les seuls fonctionnaires. Les deux lois à l'instant citées repensent la question. Celle de 2004 étend aux usagers du service public de l'éducation, les élèves dans le primaire et le secondaire, une proscription que le Conseil d'Etat n'avait pas retenue dans son avis de 1989 (sur le port du voile à l'école publique), non plus que dans sa jurisprudence subséquente. Celle de 2010, qui ne fait pas référence en tant que telle à la laïcité, va plus loin en prohibant en fait certains vêtements religieux, pour les personnes ordinaires dans l'espace même du commun - la voie publique, les commerces ou les salles de spectacle - ce que la loi appelle, de manière inédite, l'« espace public ». Il faudrait analyser aussi la loi «travail », dite El Khomri, de 2016 qui autorise désormais, en rupture avec les dispositions du droit du travail, les entreprises à proscrire les affirmations religieuses dans leur enceinte. Le législateur a, d’autre part, redéfini les motifs de 
restriction de la liberté. On fait référence ici à la notion d'ordre public, qui est une notion centrale en droit français. Elle est évoquée par les textes fondateurs de la République, tels que l'article X de la Déclaration des droits de l'homme de 1789, ou l'article $1^{\text {er }}$ de la loi de 1905. Dans les deux textes, il est prévu que la liberté s'arrête non seulement là où commence celle d'autrui, mais là aussi où elle pourrait menacer l'ordre public. Tout le problème est de savoir comment se définit l'ordre public. Initialement, la notion supporte une acception matérielle, en renvoyant aux éléments objectifs que sont la sécurité, la tranquillité et la salubrité de la société. Or, le législateur, soutenu par le Conseil constitutionnel, l’investit désormais, subrepticement, d'une valence complémentaire, immatérielle celle-là, en la rapportant de plus en plus à un modèle substantiel de comportement, lié, selon l'expression du Conseil constitutionnel dans sa décision du 7 octobre 2010, aux « exigences minimales de la vie en société »4. Ces restrictions concernent l'islam, et non les autres confessions (malgré un effet de halo). On a même vu, au cours de la période de référence, une réaffirmation des « racines chrétiennes de la France », souvent appariées à l’ordre démo-libéral.

\section{Conclusion}

Le parcours des trente dernières années nous aura confrontés à un changement décisif dans la pratique de la laïcité. La laïcité relevait, au début du XXe siècle, du registre de la norme. L' « intégrisme républicain » aurait aimé que l'Etat laïque puisse reconfigurer les cultes, et parfois même les éradiquer, dans le dessein de faire triompher au plus vite la raison. Il ne l'a pas emporté : c'est, avec Briand et Jaurès, le républicanisme libéral qui s’est imposé. La visée reste la même : il faut émanciper. La méthode est différente : elle ne restreint aucunement la liberté religieuse. Du coup, la laïcité a pris ici la figure d'un simple cadre procédural permettant à chacun de cultiver sa croyance ou son incroyance à son gré, sans que l'Etat veuille peser directement sur l'organisation et l'activité des

\footnotetext{
${ }^{4}$ Le droit de la naturalisation va dans le même sens, qui va voir l'administration et, parfois, le juge évaluer la possibilité de la naturalisation en fonction de l'adaptation du demandeur/demanderesse aux principes de la société, et notamment au principe de l'égalité des hommes et des femmes. La politique de proscription du burkini à l'été 2016 participe du même esprit.
} 
cultes. Cent-dix ans plus tard, l'Etat use autrement de la laïcité. Il en fait un instrument de reconfiguration de l'esprit public. La laïcité se construit désormais comme un dispositif de diffusion de valeurs : elle est mobilisée pour extraire les individus des communautés partielles qui, en les plaçant sous la tutelle d'une hétéronomie, font obstacle à leur accession à l'âge de leur majorité, tout en demandant aux institutions religieuses de faire valoir leurs propres convictions dans la sphère publique.

On voit ainsi s’opérer une recomposition de notre être politique. Le monde démo-libéral accorde à chacun deux identités, en situation d'extériorité l'une par rapport à l'autre : d'un côté, l'identité personnelle ou morale où résident les choix profonds de l'individu, appuyés sur des doctrines du bien ; de l'autre, l’identité politique ou institutionnelle, construite autour de droits formels, que le sujet possède en raison simplement de son appartenance à un régime constitutionnel. Le pouvoir politique n'entend nullement les confondre. Il les préserve l'une et l'autre : c'est la condition pour que le sujet puisse choisir sa propre conception du bien. Or, ce modèle est en train de se défaire, sous l'effet de « politiques transformatives » (MACEDO, 2000) axée sur la promotion de valeurs épaisses, celles d'un « libéralisme musclé »5 : le gouvernement s'emploie à substantialiser l’identité institutionnelle en même temps qu'il s'attache à reconfigurer l'identité personnelle de ses assujettis.

${ }^{5}$ Selon l'expression de David Cameron. 


\section{REFERÊNCIAS}

COC, Guy. Laïcité et République. Le lien nécessaire. Paris: Éditions du Félin, 1995.

FERRY, Jean-Marc. Valeurs et normes, La question de l'éthique. Bruxelles: Editions de l’Université de Bruxelles, 2002.

HABERMAS, Jürgen. Théorie de l'agir communicationnel. t.1, Paris: Fayard, 1987.

MACEDO, Stephen. Diversity and Distrust, Civic Education in multicultural Democracy. Cambridge: Harvard University Press, 2000.

PORTIER, Philippe. L'Etat et les religions en France. Une sociologie historique de la laïcité. Rennes: PUR, 2016.

PORTIER, Philippe. La politique du voile en France. Droits et valeurs dans la fabrique de la laïcité. Revue du droit des religions, Strasbourg, n. 2, novembre 2016.

ROMAN, Joël. Pour un multiculturalisme tempéré. Hommes \& Migrations, Paris, 1996, n. 1197, pp. 18-22.

ROY, Olivier. La laïcité à l’épreuve de l’islam. Stock: Paris, 2005. 